\title{
The impact of $\mathrm{Bi}^{3+}$ heterovalent doping in organic-inorganic metal halide perovskite crystals
}

\author{
Pabitra K. Nayak ${ }^{\mathrm{a}}$, Michael Sendner ${ }^{\mathrm{b}, \mathrm{c}}$, Bernard Wenger ${ }^{\mathrm{a}}$, Zhiping Wang ${ }^{\mathrm{a}}$, Kshama Sharma ${ }^{\mathrm{d}}$, Alexandra \\ Ramadan $^{\text {a }}$, Robert Lovrinčić ${ }^{\text {b,e }}$, Annemarie Puccib,c ${ }^{\text {b, }}$ P. K. Madhu ${ }^{\text {d,f }}$, Henry J. Snaith ${ }^{\text {* }}$. \\ ${ }^{a}$ Clarendon Laboratory, Department of Physics, University of Oxford, Oxford OX1 3PU, UK \\ bInnovationLab, Speyerer Str. 4, 69115 Heidelberg, Germany \\ ${ }^{c}$ Kirchhoff Institute for Physics, Heidelberg University, Im Neuenheimer Feld 227, 69120 Heidelberg, Germany \\ ${ }^{d}$ TIFR Centre for Interdisciplinary Sciences, 21 Brundavan Colony, Narsingi, Hyderabad, 500075, India \\ ${ }^{\mathrm{e}}$ Institute for High Frequency Technology, TU Braunschweig, Schleinizstr. 22, 38106 Braunschweig, Germany \\ fDepartment of Chemical Sciences, Tata Institute of Fundamental Research, Homi Bhabha Road, Mumbai, 400005, India \\ Supporting Information Placeholder
}

\begin{abstract}
Intrinsic organic-inorganic metal halide perovskites (OIHP) based semiconductors have shown wide applications in optoelectronic devices. There have been several attempts to incorporate heterovalent metal (e.g. $\mathrm{Bi}^{3+}$ ) ions in the perovskites in an attempt to induce electronic doping and increase the charge carrier density in the semiconductor. It has been reported that inclusion of $\mathrm{Bi}^{3+}$ decreases the bandgap of the material considerably. However, contrary to the earlier conclusions, despite a clear change in the appearance of the crystal as observed by eye, here we show that bandgap of $\mathrm{MAPbBr}_{3}$ crystals does not change due the presence of $\mathrm{Bi}^{3+}$ in the growth solution. Formation of band gap states and use of a very thick sample for transmission measurements erroneously give the impression of bandgap shift. We find that the microstrains and the density of sub-band gap states increases due to the inclusion of $\mathrm{Bi}$ in the perovskite crystal. These bandgap states also act as nonradiative recombination centers in the crystals.
\end{abstract}

Optoelectronic devices based on organic-inorganic metal halide perovskite (OIHP) have shown tremendous progress in the last few years in optoelectronic applications, owing to their high electronic quality ${ }^{1,2}$. This class of materials is often used as intrinsic semiconductors in devices ${ }^{3}$. However, almost all semiconductor materials, including GaAs, benefit from the ability to electronically dope, either p- or n-type. There have been several attempts to dope metal halide perovskite materials by incorporating isovalent (e.g. $\left.\mathrm{Sr}^{2+}, \mathrm{Cd}^{2+}, \mathrm{Sn}^{2+}, \mathrm{Ca}^{2+}\right)^{4,5,6}$ and heterovalent ions like $\left(\mathrm{Bi}^{3+}, \mathrm{Sb}^{3+}\right.$, $\left.\mathrm{In}^{3+}\right)^{7}$. Abdelhady et al. reported that heterovalent doping of methylammonium lead bromide (MAPbBr3) crystals is possible by introducing $\mathrm{Bi}^{3+}$ ions into the precursor solution during the crystal growth ${ }^{7}$. Abdelhady et al. appeared to observe a bandgap narrowing. The band gap narrowing has also been reported for the $\mathrm{CsPbr}_{3}$ and $\mathrm{MAPbCl}_{3}$ crystals with $\mathrm{Bi}^{3+}$ doping ${ }^{8,9}$ and also for $\mathrm{Cs}_{2} \mathrm{AgBiBr}_{6}$ with $\mathrm{Tl}^{+}$doping ${ }^{10}$. In OIHPs, a change in band gap is associated with the change in the crystal lattice parameters, ${ }^{10,11}$ typically inducing a change in the lead-halide octahedral tilting angle ${ }^{12}$. In the case of $\mathrm{Bi}^{3+}$ doped $\mathrm{MAPbBr}_{3}$ crystals, no such change in crystal lattice parameters has been observed ${ }^{7}$. Herein, we show that doping with $\mathrm{Bi}^{3+}$ does not change the bandgap of $\mathrm{MAPbBr} 3$, but induces a significant increase in the sub-band gap density of states, responsible for the previous misinterpretation of light absorption measurements.
We prepared the undoped and doped $\mathrm{MAPbBr} 3$ crystals using the method reported by Adbelhady and co-workers ${ }^{7}$. We confirmed the presence of $\mathrm{Bi}$ within the crystal via XPS measurements on a freshly cleaved doped single crystal (Figure S1).To avoid any ambiguity, we refer to the doped crystals with the concentration of $\mathrm{Bi}^{3+}$ salt in the growth solution. As previously observed, the prepared crystals appear darker as the concentration of $\mathrm{Bi}^{3+}$ is increased in the growth solution (Figure 1a). We reproduce the shift in the "onset" published by Abdelhady et al. ${ }^{7}$ when estimating the absorbance of the crystals from light transmission measurements (Figure 1b). As we explain hereafter, the absorption onset should not be mistakenly identified as the band gap of the material. Due to the thickness of the single crystals (several $\mathrm{mm}$ ) and the large above band gap absorption coefficients for OIHP, there is an undetectable amount of light transmitted right across the light absorbing region of the crystal, until an energy significantly below the band gap energy of the material. Therefore, these simple light transmission measurements are in fact probing the sub band gap absorptions far in the band tail ${ }^{13}$. It is therefore not possible to draw any conclusions about the band gap from such a measurement alone.
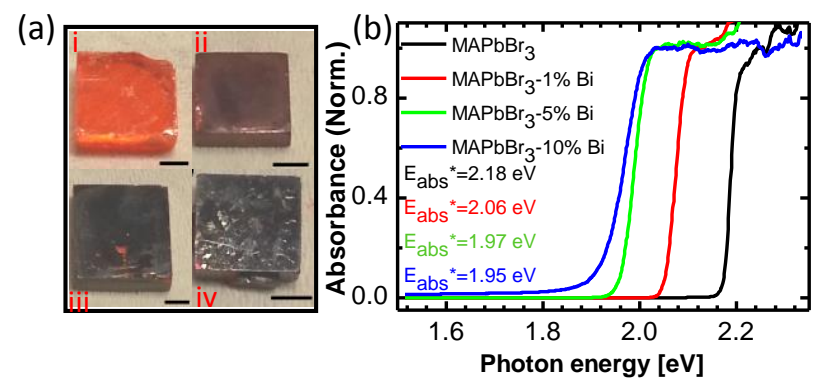

Figure 1. (a) Picture of doped and undoped $\mathrm{MAPbBr}_{3}$ single crystals. (i) pure $\mathrm{MAPbBr}_{3}$, (ii) $\mathrm{MAPbBr}_{3}$ doped with $1 \% \mathrm{Bi}^{3+}$, (iii) $\mathrm{MAPbBr}_{3}$ doped with $5 \% \mathrm{Bi}^{3+}$ and (iv) $\mathrm{MAPbBr}_{3}$ doped with $10 \%$ $\mathrm{Bi}^{3+}$. Scale bar: $2 \mathrm{~mm}$. (b) Normalized absorbance spectra of the crystals. Eabs $^{*}=$ absorption onset

Spectroscopic ellipsometry is an effective tool to estimate the above band gap optical properties of OIHP crystals ${ }^{13}$. Here, we perform such measurements upon our range of $\mathrm{Bi}^{3+}$ doped $\mathrm{MAPbBr}_{3}$ crystals. We show the measured and modeled ellipsometry spectra in Figure S2. We fitted those spectra using the Elliot model ${ }^{14}$ to 
estimate the exciton binding energy $\left(R_{e x}\right)$ and the bandgap $\left(E_{g}\right)$ from the continuum part of the spectrum. In Figure 2a, we show the experimentally determined absorption coefficients for the MAP$\mathrm{bBr}_{3}$ crystal and the crystal doped with $5 \% \mathrm{Bi}^{3+}$ as well as the fittings using the Elliot model. The step function for the continuum part and delta function for the excitonic transition are broadened by convolution with Gaussian functions, $\sigma_{\text {cont. and }} \sigma_{\text {ex }}{ }^{13}$. In Figure S3 we provide the graphs for all the crystals, and in Table S2, we provide the values of $E_{g}, R_{e x}, \sigma_{\text {cont. }}$ and $\sigma_{\text {ex }}$. We find that the $E_{g}$ values of all crystals are almost constant in the range $2.383 \mathrm{eV}$ to 2.389 $\mathrm{eV}$. The change we see is within the error of our fittings which is \pm $15 \mathrm{meV}$, hence we conclude that there is no significant change in the bandgap of the material after the $\mathrm{Bi}^{3+}$ doping. However, we note that the broadening factors $\left(\sigma_{\mathrm{cont}}\right.$ and $\left.\sigma_{\mathrm{ex}}\right)$ do increase after the doping. The broadening could be an indication of increased energetic disorder present in the system.
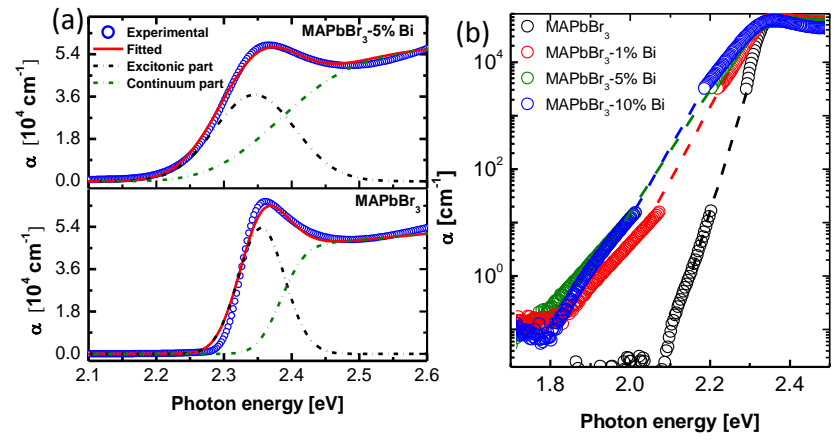

Figure 2. (a) Absorption spectra in the band edge region for a MAP$\mathrm{bBr}_{3}$ single crystal (bottom) and a $\mathrm{MAPbBr}_{3}$ single crystal with $5 \%$ Bi doping (top). (b) Combined absorption spectra from ellipsometry data (top part) and the UV-vis transmission data (bottom part). The dashed lines are the guides to the eye only.

We also determine the bandgap of the crystals using Tauc-plot method for a direct bandgap material. The band gap seems to decrease by $30 \mathrm{meV}$ (Figure S4) on doping

Our observation of negligible change in the band gap is in contrast to reported values in the literature ${ }^{7}$, where a reduction of the bandgap of $280 \mathrm{meV}$ was estimated from the absorption spectra measured in light transmission mode. As we mentioned earlier, the band gap estimated from the normalized absorbance plot of strongly absorbing single crystals (Figure 1) can be erroneous due to the long optical path in a thick crystal. To correctly represent the absorption spectra, we combine the absorption coefficients $(\alpha)$ obtained from ellipsometry and $\alpha$ calculated from total transmittance $T_{T}$ and total reflectance $R_{T}$. (Figure $2 \mathrm{~b}$ ). As we show in Figure 2b, although the bandgap remains the same, the absorption tail increases at lower energies with the presence of $\mathrm{Bi}^{3+}$ in the growth solution. The exponential slope in the sub-band gap density of states, quantified by the Urbach energy $\left(\mathrm{E}_{\mathrm{u}}\right)$, can be used as metric to gauge the energetic disorder in the crystal. For the pristine crystal, we determine an Urbach energy of $\sim 19 \mathrm{meV}$ compared to 50$55 \mathrm{meV}$ that of the doped crystals. To further confirm, we prepared $\mathrm{Bi}^{3+}$ doped thin-films of $\mathrm{MAPbBr}_{3}$ and measured the absorbance spectra. We see no significant change in the absorption onset values (Figure S5).

We note that several works claiming a band gap narrowing of halide perovskites due to doping have utilized diffuse reflectance measurements for the band gap determination ${ }^{10,15}$. However, diffuse reflectance measurements from powdered samples are also subject to the same errors as transmission measurements ${ }^{16}$.
To investigate the effect of the presence of $\mathrm{Bi}^{3+}$ on the lattice vibrations of $\mathrm{MAPbBr}_{3}$, we performed Far-IR measurements on the crystals. In Figure S6 we show the resulting reflection spectra for the undoped and $\mathrm{Bi}$-doped $\mathrm{MAPbBr} 3$ crystals. The abrupt reflectance feature we observe in this region of the spectrum, is due to the frequency of light being close to the vibrational frequency of the ions in the crystal, which prevents the propagation of light through the crystal at this frequency. We observe here to be very similar for the three investigated single crystals, and in agreement with a recent publication ${ }^{17}$. We further analyzed the spectra by using a model based on a Gervais oscillator ${ }^{17,18}$. We do not determine significant differences between the fitted resonance frequencies nor the damping constant, as we show in Table S3. As there is no change in the far IR features due to the doping, we anticipate that the upper limit for mobility at the room temperature still remains the same i.e. $\sim 200 \mathrm{~cm}^{2} / \mathrm{V} . \mathrm{s}^{17}$. Moreover, the presence of the band gap states due to Bi doping would reduce the mobility value further. Therefore, a mobility value of $1250 \mathrm{~cm}^{2} / \mathrm{Vs}$ determined by Abdelhady et al. for the doped crystals is put into question (See SI)

To further analyze the influence of the $\mathrm{Bi}^{3+}$ doping we examined the molecular vibrations of the crystals in the mid-infrared spectral range, by means of spectroscopic ellipsometry. We included the optical parameters which we determined in the far-infrared spectral range, into the optical model based on Glaser et al. ${ }^{19}$ We show the optical fit of the ellipsometry spectra with an isotropic model in Figure S7 along with the resulting dielectric functions of the undoped $\mathrm{MAPbBr}_{3}$ and the $10 \% \mathrm{Bi}^{3+}$ doped $\mathrm{MAPbBr}_{3}$ single crystal. We observe a very similar imaginary part of the dielectric function for the highest doped crystal and the undoped crystal. The vibrations are excited at the same energies as in the undoped case. We notice a spectral broadening in the region of the $\mathrm{NH}$ stretching vibrations around $3200 \mathrm{~cm}^{-1}$ in the doped sample (Figure S8), which indicates a higher disorder since these vibrations are very sensitive to the dielectric background ${ }^{19,20}$.
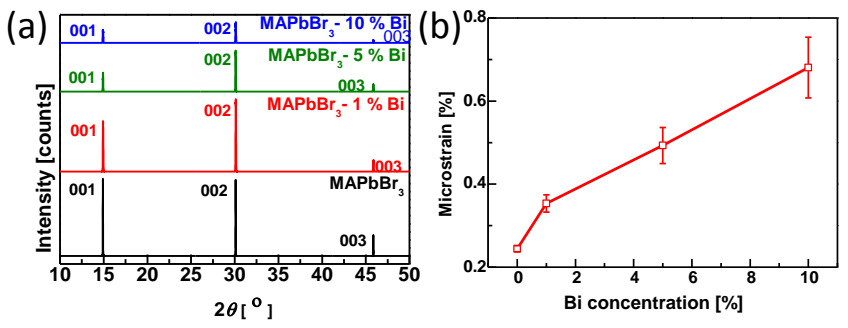

Figure 3. (a) XRD patterns of undoped and $\mathrm{Bi}$ doped $\mathrm{MAPbBr}_{3}$ crystals, (b) Calculated microstrain of samples with different concentrations of Bismuth doping.

To identify the impact of Bismuth incorporation on the crystal structure of $\mathrm{MAPbBr}_{3}$ single crystals, we performed X-ray diffraction (XRD) measurement. In Figure $3 a$ we show the $2 \theta / \omega$ scans of the pristine $\mathrm{MAPbBr}_{3}$ single crystal and $\mathrm{MAPbBr}_{3}$ single crystals with different $\mathrm{Bi}^{3+}$ doping concentrations. The average $\mathrm{Bi}-\mathrm{Br}$ bond length in $\mathrm{BiBr}^{3-}{ }^{3-}$ octahedra $(2.87 \AA$, calculated from the axial and equatorial bonds ${ }^{21}$ ) is very close to the average $\mathrm{Pb}-\mathrm{Br}$ bond length $(2.97 \AA)$ in a $\mathrm{MAPbBr}_{3}$ single crystal ${ }^{22}$. Though we expect a very small change in the in the lattice parameter due to the incorporation of $\mathrm{Bi}$, we do not determine a resolvable shift in the XRD peaks in our measurement (Figure S9) or see any new diffraction peaks in the XRD patterns due to doping.

We hypothesize that the Bismuth is uniformly distributed throughout the crystal, but its presence, either in the final crystals or simply during the crystallization process, is primarily disrupting the crystalline order and increasing the electronic disorder, rather than resulting in a systematic shift in the crystal structure. The 
reduced crystalline order may be detectable in the form of nonuniform strain (microstrain) in the $\mathrm{MAPbBr}_{3}$ crystals, induced by crystal imperfections/structural defects including dislocations, vacancies, stacking faults, etc. ${ }^{23}$. We quantify the extent of microstrain in our perovskite crystals by analyzing the peak broadening in the diffraction patterns according to the modified Williamson-Hall method (see SI).$^{23,24}$ Consistently, we determine the microstrain in the crystals to increase upon doping (Figure $3 b$ and Figure S10). Furthermore, we observe gradual broadening in the rocking curves ( $\omega$ scans) of crystals with increasing doping (Figure S11) which indicates distortions in the crystal planes across the crystals.

In order to obtain information about the uniformity of the chemical environment around the $\mathrm{Pb}^{2+}$ center and the protons of methylammonium, we performed solid-state ${ }^{207} \mathrm{~Pb}$ NMR and ${ }^{1} \mathrm{H}$ NMR of the crystals. In Figure $4 \mathrm{a}$, we show the ${ }^{207} \mathrm{~Pb}$ NMR spectra of the undoped and doped crystals. We observe that the NMR spectrum is broadened for the doped crystal, implying a variable environment around the $\mathrm{Pb}$ and $\mathrm{MA}$ ions. For the ${ }^{207} \mathrm{~Pb}$ centers, the broadening is probably due to the increased disorder in the crystal as the chemical shift of nuclei is sensitive to the local structural distortions ${ }^{25}$. We also see a similar broadening of the ${ }^{1} \mathrm{H}$ NMR spectrum (Figure S12)
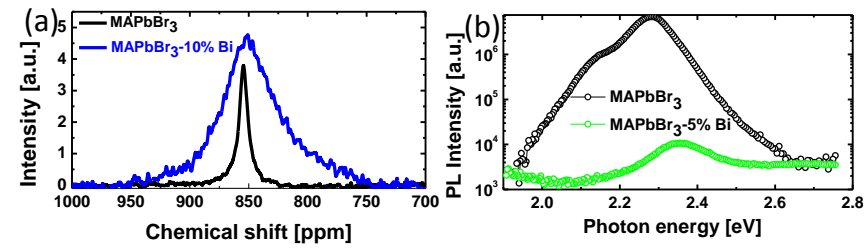

Figure 4. (a) ${ }^{207} \mathrm{~Pb}$ NMR spectrum of the undoped and Bi-doped $\mathrm{MAPbBr} 3$ crystals. (b) Steady-state PL spectrum of $\mathrm{MAPbBr}_{3}$ and Bi-doped MAPbBr 3 .

After establishing the impact of bismuth doping on the energetic disorder and microstrains, we looked for the impact on the photoluminescence (PL) properties of the materials. In Figure $4 \mathrm{~b}$ we show the PL spectrum of the crystals and the time-resolved PL decays in Figure S13. We find that the PL intensity is quenched by $>99 \%$ in the doped crystals. We also observe a lowering of the average PL decay lifetime in the presence of $\mathrm{Bi}^{3+}$ doping, via timeresolved photoluminescence (TRPL) decay measurements. All our findings are consistent with the presence of bismuth introducing defect states in the crystal, which facilitating unfavorable non-radiative recombination, thereby lowering the PL intensity and PL lifetime.

To understand the effect of doping on the charge transport, we prepared solar cells with the following configuration FTO/SnO $/ \mathrm{MAPbBr}_{3}$ (Bi-doped)/Spiro-OmeTAD/Ag. We measured the EQE of the devices and present the data in the Figure S14. We find EQE drops with increased concentration of $\mathrm{Bi}$ in the MAP$\mathrm{bBr}_{3}$ films. This finding indicates that the introduction Bi introduces traps in the thinfilm which in turn reduces the carrier collection efficiency. In addition, we do not see any shift in the onset of the EQE spectrum which reaffirms our conclusion that the introduction of $\mathrm{Bi}$ does not change the bandgap of the material

The estimation of the bandgap from light transmission measurements can be difficult due to the strong absorption coefficients and the thickness of the crystals ${ }^{13}$. Previous reports on $\mathrm{Bi}^{3+}$ doping of OIHP have underestimated this effect, and have thus reported band gap reductions that we can't reproduce when using appropriate measurement techniques such as ellipsometry. We note that such erroneous band gap shifts have been also reported for undoped perovskite single crystals or thick polycrystalline samples in several studies published in leading journals (see SI). The origins of such (apparent) band gap shifts (reported to be an unsolved problem ${ }^{26,27}$ ) can be explained considering the band edge tail absorption in thick samples ${ }^{13}$ while the band gap value remains constant.

From our experimental work, we are now able to provide a more detailed picture of the doping of $\mathrm{MAPbBr}_{3}$ single crystals by bismuth. The absence of band gap reduction, combined with an increased energetic disorder points towards the introduction of defects in the perovskite by the presence of $\mathrm{Bi}^{3+}$ ions in the growth solution. In addition, the strong quenching of the photoluminescence and decrease in EQE of indicates that the electronic states introduced by the dopants are detrimental to the optoelectronic quality of the material.

In conclusion, we have shown that bandgap narrowing does not occur in the $\mathrm{MAPbBr} 3$ crystals following the incorporation of $\mathrm{Bi}^{3+}$ in the growth solution. The apparent effect of the colour change, is due to the increased number of defect states and a significant increase in the sub-band gap density of states. The Bi-doped crystals have higher levels of microstrain, increased electronic disorder and reduced carrier lifetime and PL efficiency, due to increased nonradiative recombination. Our study highlights the challenges with heterovalent doping, particularly with $\mathrm{Bi}$, of OIHP semiconductors. More studies with different dopants will gauge the usefulness of such an approach in order to control the structural and electronic properties of OIHP.

We finally note that a recent publication identified bismuth doping of $\mathrm{CsPbI}_{3}$ as a feasible route to increase the structural stability, induce a $174 \mathrm{meV}$ lowering of the absorption edge, and increase the PL efficiency and photovoltaic solar energy conversion efficiency ${ }^{28}$. This recent publication is inconsistent with our findings here, and indicates that further work is still required to understand the impact of heterovalent doping upon OIHP.

\section{Supporting Information}

\section{AUTHOR INFORMATION}

Corresponding Author: Henry.Snaith@physics.ox.ac.uk

\section{ACKNOWLEDGMENT}

This work is supported by the EPSRC UK. MS and RL acknowledge the InterPhase project from BMBF (FKZ 13N13656, 13N13657). BW acknowledges Marie Skłodowska-Curie actions (706552-APPEL). We thank Sebastian Beck and Nobuya Sakai for their assistance in UV-vis absorption measurements.

\section{REFERENCES}

(1) Manser, J. S.; Christians, J. A.; Kamat, P. V. Chem. Rev. 2016, $116(21), 12956$.

(2) Stranks, S. D.; Snaith, H. J. Nat. Nanotechnol. 2015, 10 (5), 391.

(3) Edri, E.; Kirmayer, S.; Mukhopadhyay, S.; Gartsman, K.; Hodes, G.; Cahen, D. Nat. Commun. 2014, 5 , ncomms 4461.

(4) Klug, M. T.; Osherov, A.; Haghighirad, A. A.; Stranks, S. D.; Brown, P. R.; Bai, S.; Wang, J. T.-W.; Dang, X.; Bulović, V.; Snaith, H. J.; Belcher, A. M. Energy Environ. Sci. 2017, 10 (1), 236.

(5) Lau, C. F. J.; Zhang, M.; Deng, X.; Zheng, J.; Bing, J.; Ma, Q.; Kim, J.; Hu, L.; Green, M. A.; Huang, S.; Ho-Baillie, A. ACS Energy Lett. 2017, 2319.

(6) Hao, F.; Stoumpos, C. C.; Chang, R. P. H.; Kanatzidis, M. G. J. Am. Chem. Soc. 2014, 136 (22), 8094.

(7) Abdelhady, A. L.; Saidaminov, M. I.; Murali, B.; Adinolfi, V.; Voznyy, O.; Katsiev, K.; Alarousu, E.; Comin, R.; Dursun, I.; 
Sinatra, L.; Sargent, E. H.; Mohammed, O. F.; Bakr, O. M. J. Phys. Chem. Lett. 2016, 7 (2), 295.

Miao, X.; Qiu, T.; Zhang, S.; Ma, H.; Hu, Y.; Bai, F.; Wu, Z. J. Mater. Chem. C 2017, 5 (5), 4931.

(9) Zhang, Z.; Ren, L.; Yan, H.; Guo, S.; Wang, S.; Wang, M.; Jin, K. J. Phys. Chem. C 2017, 121 (32), 17436.

(10) Slavney, A. H.; Leppert, L.; Bartesaghi, D.; Gold-Parker, A.; Toney, M. F.; Savenije, T. J.; Neaton, J. B.; Karunadasa, H. I. J. Am. Chem. Soc. 2017, 139 (14), 5015.

(11) Eperon, G. E.; Stranks, S. D.; Menelaou, C.; Johnston, M. B.; Herz, L. M.; Snaith, H. J. Energy Environ. Sci. 2014, 7 (3), 982.

(12) Filip, M. R.; Eperon, G. E.; Snaith, H. J.; Giustino, F. Nat. Commun. 2014, 5, 5757.

(13) Wenger, B.; Nayak, P. K.; Wen, X.; Kesava, S. V.; Noel, N. K.; Snaith, H. J. Nat. Commun. 2017, 8 (1), 590.

(14) Elliott, R. J. Phys. Rev. 1957, 108 (6), 1384.

(15) Du, K. Z.; Wang, X.; Han, Q.; Yan, Y.; Mitzi, D. B. ACS Energy Lett. 2017, 2 (10), 2486.

(16) Murphy, A. B. Sol. Energy Mater. Sol. Cells 2007, 91 (14), 1326

(17) Sendner, M.; Nayak, P. K.; Egger, D. A.; Beck, S.; Müller, C.; Epding, B.; Kowalsky, W.; Kronik, L.; Snaith, H. J.; Pucci, A.; Lovrinčić, R. Mater. Horiz. 2016, 3 (6), 613.

(18) Gervais, F.; Piriou, B. J. Phys. C Solid State Phys. 1974, 7 (13), 2374.

(19) Glaser, T.; Müller, C.; Sendner, M.; Krekeler, C.; Semonin, O.
E.; Hull, T. D.; Yaffe, O.; Owen, J. S.; Kowalsky, W.; Pucci, A.; Lovrinčić, R. J. Phys. Chem. Lett. 2015, 6 (15), 2913.

(20) Müller, C.; Glaser, T.; Plogmeyer, M.; Sendner, M.; Döring, S.; Bakulin, A. A.; Brzuska, C.; Scheer, R.; Pshenichnikov, M. S.; Kowalsky, W.; Pucci, A.; Lovrinčić, R. Chem. Mater. 2015, 27 (22), 7835.

(21) Bi, W.; Leblanc, N.; Mercier, N.; Auban-Senzier, P.; Pasquier, C. Chem. Mater 2009, 21, 4099.

(22) Rakita, Y.; Cohen, S. R.; Kedem, N. K.; Hodes, G.; Cahen, D. MRS Commun. 2015, 5 (4), 623.

(23) Pramanick, A.; Wang, X. P.; Hoffmann, C.; Diallo, S. O.; Jørgensen, M. R. V.; Wang, X.-L. Phys. Rev. B 2015, 92 (17), 174103.

(24) Zhao, Y.; Zhang, J.; IUCr. J. Appl. Crystallogr. 2008, 41 (6), 1095.

(25) Brouwer, D. H.; Brouwer; H., D. In Encyclopedia of Magnetic Resonance; John Wiley \& Sons, Ltd: Chichester, UK, 2008.

(26) Huang, J.; Yuan, Y.; Shao, Y.; Yan, Y. Nat. Rev. Mater. 2017, 2 (7), 17042.

(27) Adinolfi, V.; Peng, W.; Walters, G.; Bakr, O. M.; Sargent, E. H. Adv. Mater. 2017, 1700764.

(28) Hu, Y.; Bai, F.; Liu, X.; Ji, Q.; Miao, X.; Qiu, T.; Zhang, S. ACS Energy Lett. 2017, No. 2, 2219.

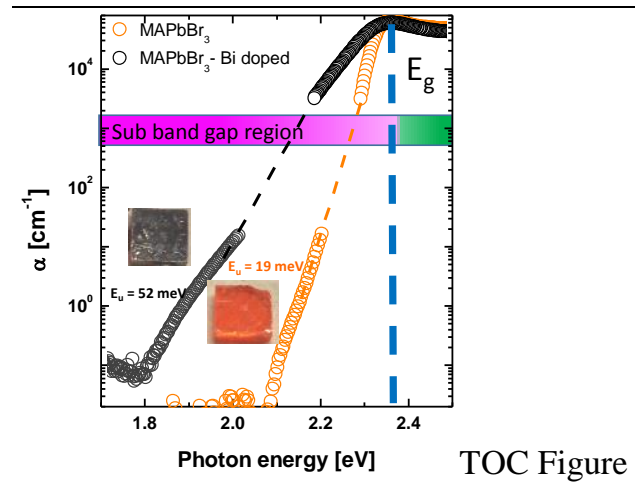

